CORRIGENDUM

doi:10.1038/nature08360

\title{
CBP/p300-mediated acetylation of histone H3 on lysine 56
}

Chandrima Das, M. Scott Lucia, Kirk C. Hansen \& Jessica K. Tyler

\section{Nature 459, 113-117 (2009)}

In Fig. 4e of this Letter, one band was incorrectly labelled. We thank B. Lüscher for drawing this to our attention. The correctly labelled version of the figure is provided below. As such, the statement that there is "a notable correlation between levels of H3K56 acetylation and ASF1A but not ASF1B in a wide variety of normal and cancerous human tissues" is no longer accurate. However, the main purpose of Fig. $4 \mathrm{e}$ is still valid, which was to show by western blotting analysis that the level of H3K56ac is much higher in some tumours as compared to the matching normal tissue.

We also note that although the fractionation of the total stained proteins in Figs 1f and $4 \mathrm{~d}$ did not show obvious differences, the fractionation of histones and tubulin was effective between the supernatant and pellet fractions (see Supplementary Figure).

We also omitted to state that the ultraviolet light used in our analysis was $312 \mathrm{~nm}$ UVB, which causes $1 \%$ of the amount of DNA damage as $254 \mathrm{~nm}$ UVC.

Supplementary Information is linked to the online version of the paper at www.nature.com/nature.

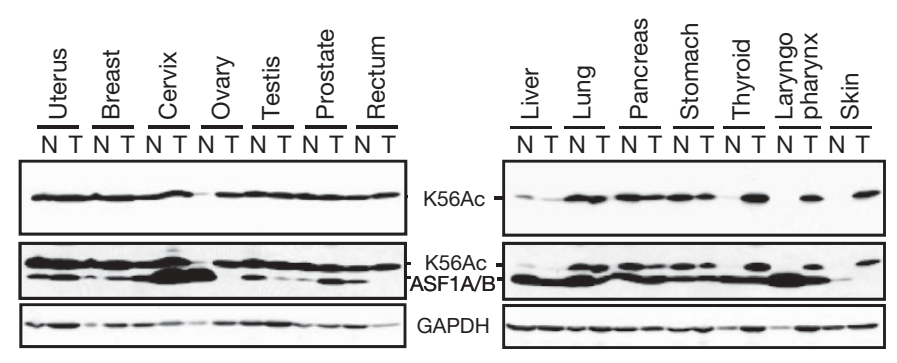

